\title{
Automated Imaging System for Pigmented Skin Lesion Diagnosis
}

\author{
Mariam Ahmed Sheha/ Amr Sharwy \\ Biomedical and system Engineering \\ Cairo University \\ Giza, Egypt
}

\author{
Mai S. Mabrouk* \\ Biomedical Engineering Dept. \\ MUST University \\ Giza, Egypt
}

\begin{abstract}
Through the study of pigmented skin lesions risk factors, the appearance of malignant melanoma turns the anomalous occurrence of these lesions to annoying sign. The difficulty of differentiation between malignant melanoma and melanocytic naive is the error-bone problem that usually faces the physicians in diagnosis. To think through the hard mission of pigmented skin lesions diagnosis different clinical diagnosis algorithms were proposed such as pattern analysis, ABCD rule of dermoscopy, Menzies method, and 7-points checklist. Computerized monitoring of these algorithms improves the diagnosis of melanoma compared to simple naked-eye of physician during examination. Toward the serious step of melanoma early detection, aiming to reduce melanoma mortality rate, several computerized studies and procedures were proposed. Through this research different approaches with a huge number of features were discussed to point out the best approach or methodology could be followed to accurately diagnose the pigmented skin lesion. This paper proposes automated system for diagnosis of melanoma to provide quantitative and objective evaluation of skin lesion as opposed to visual assessment, which is subjective in nature. Two different data sets were utilized to reduce the effect of qualitative interpretation problem upon accurate diagnosis. Set of clinical images that are acquired from a standard camera while the other set is acquired from a special dermoscopic camera and so named dermoscopic images. System contribution appears in new, complete and different approaches presented for the aim of pigmented skin lesion diagnosis. These approaches result from using large conclusive set of features fed to different classifiers. The three main types of different features extracted from the region of interest are geometric, chromatic, and texture features. Three statistical methods were proposed to select the most significant features that will cause a valuable effect in diagnosis; Fisher score method, t-test, and F-test. The selected features of high-ranking score based on the statistical methods are used for the diagnosis of the two lesion groups using Artificial Neural Network (ANN), K-Nearest Neighbor (KNN) and Support Vector Machine (SVM) as three different classifiers proposed. The overall System performance was then measured in regards to Specificity, Sensitivity and Accuracy. According to the different approaches that will be mentioned later the best result was showen by the ANN designed with the feature selected according to fisher score method enables a diagnostic accuracy of $96.25 \%$ and $97 \%$ for dermoscopic and clinical images respectively.
\end{abstract}

Keywords-component; Pigmented Skin lesions; Color Space; Bounding box; local range; SVM; local range; KNN; ANN; Experimental models

\section{INTRODUCTION AND LITERATURE REVIEW}

Patients seeking for dermatologic consultation are steadily increasing worldwide. The most critical type of skin diseases is melanoma, which represents the malignant type of skin cancer. Malignant melanoma usually has slow growth rate the time if the lesion detected in this early stage, it can be easily removed with a relatively low cost and hazard. Thus, the survival rate increased to 95\% if detected early. While, progression of the disease in the late stage is associated with poor survival rate of $13 \%$ [1].

For that reason, melanoma detection weighted as a challenging problem for dermatologist. Several techniques and procedure were proposed for the purpose of melanoma diagnosis; such as dermoscopy or Epiluminescence (ELM) [2], which was entertained with high expectations inspecting skin lesions. ELM was super promising as it is an in-vivo, noninvasive technique used for structures visualization enhancement under the skin surface by means of oil immersion and magnifying instrument, called a “dermatoscope"[3]. However, dermoscopic images results a magnified view for skin lesions its interpretation and diagnosis accuracy mainly depends on the experience of the viewer. Several diagnosis models with similar reliability have become more widely accepted by physicians [4,5] as, 7-point check, Menzie rule and the most popular scoring system so called $\mathrm{ABCD}$ rule.

Determination and extraction of features that mention lesion characteristics are usually the error-prone operation through all automated diagnosis systems. Thus, introducing these automated diagnosis systems, as a non-invasive diagnosis support tool is endless interesting work. A considerable number of resaeches and publications focused on the area of image analysis and pattern classification related to melanoma images identification and classifications. Over the last 20 years, the computer aided diagnosis systems for melanoma diagnosis developed [6] to have diagnosis accuracy around $73 \%$ to $98 \%$.

July 2002, P. Rubegni et al. [7] developed an automated diagnosis system for melanoma with a set of 50 objective features regarding the main three categories of features that could accurately describe the malignancy of lesions; geometries, textures and islands of color. Thirteen significant features were selected, that discriminate the 2 groups of 
lesions reaching 94\% accuracy using an ANN for classification. By 2006, Iris Cheng designed an algorithm using relative color features; the algorithm was tested upon 80 nevus, 160 melanoma and 42 dysplastic nevi, as a database. Result showed [8] the algorithm ability to classify $86 \%$ of the malignant melanomas successfully in comparison with approximately $75 \%$ success rate of dermatologists.

By the beginning of 2009, José Fernández Alcón, et al proposed efficient automated system [9] for diagnosis of PSL. The system supports conventional clinical images for skin lesions acquired using digital cameras (Consumer level), in addition to a decision support component. The use of decision support component system was significant; it combine the image classification outcome with context knowledge such as skin type, age, gender, and affected body part. Lesions were classified with a sensitivity of $94 \%$, specificity of $68 \%$ and accuracy $86 \%$.

Mariam A.Sheha, et al. works toward improving contributions about the essential characteristics of PSL. By March 2010, they proposed [10] a Computer aided diagnosis system (CAD) that mainly concerned by analyzing only dermoscopic images based on gray level Co-occurrence matrix (GLCM) classified by Multilayer perceptron (MLP) to discriminate between melanocytic nevi and malignant melanoma. Moreover it was interesting to study the effect of this system using clinical images and compare results with dermoscopic images that was clearly represented on 2013 [11]. Other different features and classifiers were widely used in all fields of automated analysis and diagnosis that was valuable to introduce and study its effect. On Dec 2014, the effect of morphological features (Geometric and Chromatic) was handled using two different classifiers ANN and SVM [12].

To cope with the development track, this paper presented as a completion for the prior studies. Whereas, its main aim is to design a fully automated computer aided diagnosis system for PSL to increase system flexibility and efficiency. It concerns about different types and a huge number of features to evaluate the efficiency of each upon melanoma diagnosis. Feature extraction is usually based on two different concepts, namely morphological (geometric, chromatic) and texture analysis. Significant features that can accurately describe melanoma were then selected by three different methods of feature selection f-test, t-test and fisher score ranking. Afterwards; the study introduced three classifiers to discriminate between malignant melanoma and benign nevi regarding the selected parameters.

The following sections will mention the system performed for the digital image analysis; system designed using different analytical parameters and classification methods performing different approaches. This paper will be organized as shown; First section presented a brief introduction about the topic and related works. Then, material section will deal with the database and the software tool used in this study. By third section, the work methodology will be discussed in details including main block diagram and different algorithms applied to propose a proper and efficient diagnosis approach for PSL diagnosis. The results of the constructed approaches were compared to investigate the more suitable approach for melanoma diagnosis through the experimental setup in the fourth section. Finally; Conclusion and expected future work conclusion submitted.

\section{MATERIAL}

\section{A. Database}

Toward productive screening process various noninvasive, in-vivo imaging technique proposed. The imaging modalities used nowadays could also facilities skin lesion structure visualization such as, Positron Emission Tomography (PET), Dermoscopy, Confocal laser scanning microscopy (CLSM), High frequency ultrasound, Optical coherence tomography (OCT) and Magnetic Resonance Imaging (MRI) [13]. The study proposed was applied upon a long time used data as a set of traditional photography, named by clinical images and another set of dermoscopic images [14, 15].

The main aim through this research is to define the effective characteristics for designing a high performance consumer application CAD system with high prediction accuracy. So that, it was recommended to apply the same algorithm on both types of data sets mentioned to caliper the effect of different imaging techniques upon diagnosis.

Database presented through this study consist of 320 images for different pigmented skin lesions. Data were randomly sampled from the skin diseases atlases and doctors“ clinics [16,17]. Images were fairly divided to the two basic database types; 160 lesions for each dermoscopic and clinical. To compare between malignant and benign lesion each of the used databases were fairly divided also to 80 lesion of each group. Majority of the dermoscopic images were randomly sampled from [18] as the author of [19] recommended, clinical images used for that purpose are widely available in [20-24].

\section{B. Implementation}

For successful diagnosis and high performance automated system a good visualization is essential. Leveraging the rich graphics functionality of MATLAB, system was implemented and tested on the WINDOWS platform with MATLAB version of 2010 .

\section{METHODOLOGY}

The main scope of this paper is to apply different and new features on a computer-based system forming different experimental approaches. For that purpose it is valuable to detect the region of interest so the PSL can be accurately evaluated. Once the lesion boundary had been identified, the program attempts to extract different features to manipulate their importance in that field. The selected features are classified to assess the accuracy degree and evaluate the performance of that approach. The features used with the most accurate approach will be highly recommended as a scientific characteristic to recognize dermoscopic. Fig. 1, outlines the procedure followed by the different approaches used to diagnose clinical and dermoscopic images for PSL. 


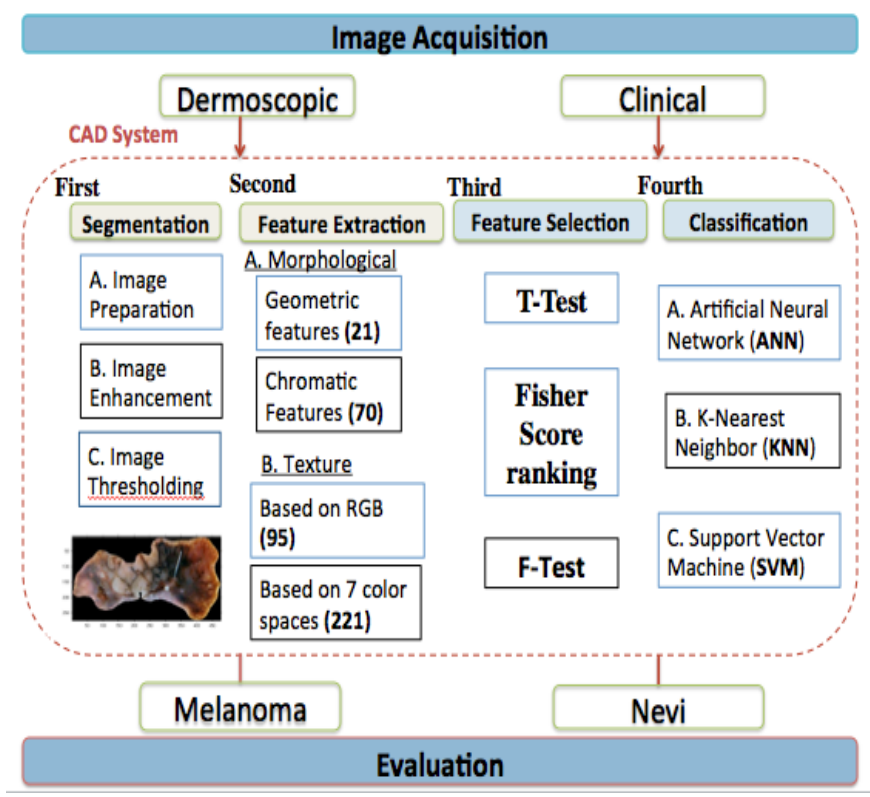

Fig. 1. The Main block diagram

\section{A. Image Segmentation}

Skin lesion images, especially clinical and dermoscopic images are often not clear and altered by uneven illumination. Typical skin artifact as hair, skin structures, oil bubbles [25] are usually considered as the main cause for such illumination, which have a direct effect on both segmentation and feature extraction algorithms. Image segmentation is an essential preprocessing step that copes with skin lesions irregularities and artifacts. Sequentially, it is necessary for efficient feature extraction and accurate classification. Thus, image preparation, enhancement and thresholding are considered as the main processing steps for segmentation.

a) Image Preparation: This step deals with scaling all images to one scale $(470 * 640)$ pixels to avoid the wide size variation in the selected database.

b) Images Enhancement: Segmentation can be distracted by the presence of skin artifacts. Two successive steps are necessery to increase the tumor border visibility and ensure its correct identification. First is image filteration using median filter [26]; considered as a correction tool for reducing noise that corrupt image's clarity. Although this procedure ends in relatively blurring of the image as shown in Fig. 2, it assists the segmentation procedure. Second step is gray level conversion; the RGB colored image is converted into intensity or gray scale image [27], To reduce the effect of different skin color variations and background.

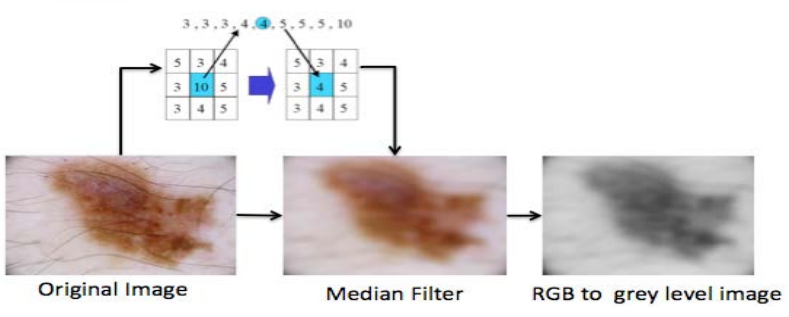

Fig. 2. Image Enhancement process

c) Images Thresholding: The common purpose of segmentation is to group the homogeneous pixels into regions with respect to specific features and semantic content. Segmentation step through this research aims to extract the lesion border from the healthy skin [28]. For accurate segmenation process and correct detection for lesion boundary, image thrsholding step should follow contrast adjustment. Through contrast or intensity adjustment, image is adjusted by saturating $1 \%$ of the pixels at low and high intensities of the output image. Fig. 3 shows the effect of adjustment process upon the filtered intensity image.

Afterwards; image thresholding process successively occured. It mainly depends on Otsu's method [29], where it labels each pixel of the entire image as belonging to the lesion or to the background. Thresholding idea can be easily mentioned by seperating the pixels whoes intensity value is below specific threshold from others that exceeds the same threshold. Then, label each of the two groups according to their definition. Through this reasearch, pixels with low intensity value are considered as the region of interest. Fig. 4 shows the conversion happened as a result of Otsu's threshold.

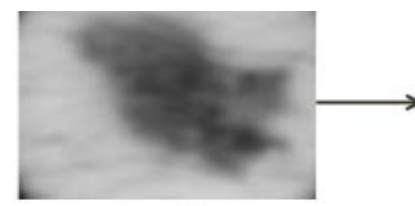

Gray Level Image

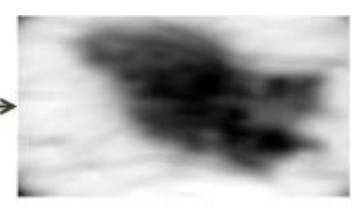

Intensity adjusted
Fig. 3. Image contrast adjustment

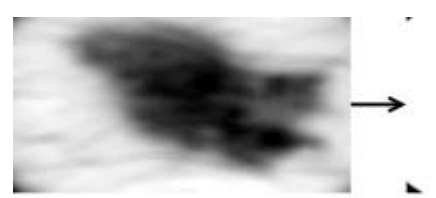

Intensity Adjustment

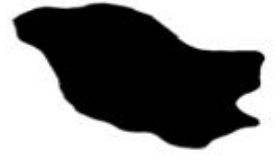

Threshold image
Fig. 4. Threshold process 
After the threshold process, it is supposed that segmentation process is almost finished, but important three accomplishment steps still remains, namely for accurate tumor detection. By means of morphological operations [30], an image result from thresholding step was processed. First, any object with size less than 150 pixels were removed. Then, Binary image is inverted to refer for the selected object by white pixels "ones" and black pixels "zero" for background. Finally, any gap that may found at lesion mask was filled to complete tumor holes. After the tumor had been segmented, lesion center (centroid) and bounding box is determined regarding the traced boundary [31]. Fig. 5. Illustrate the full segmentation process.

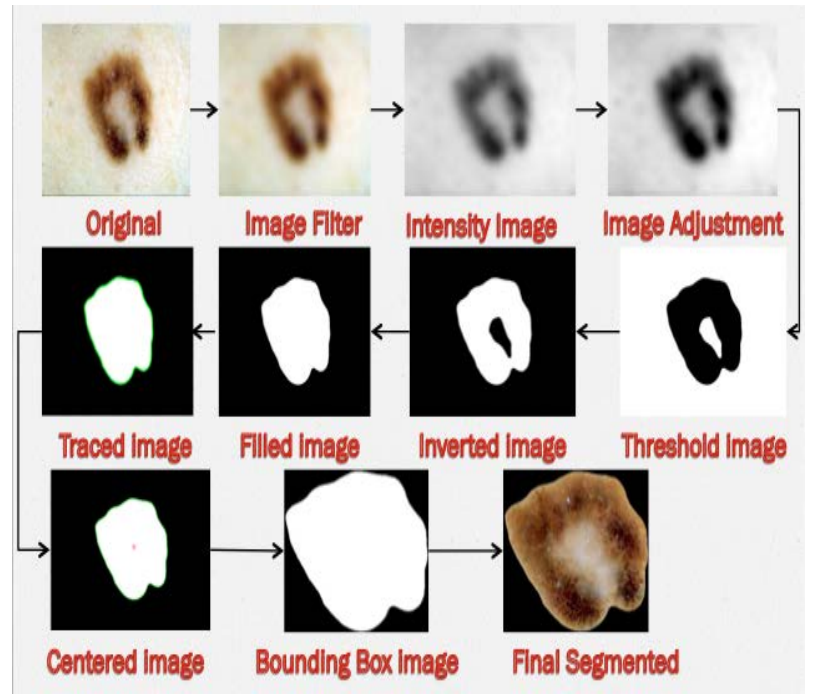

Fig. 5. Schematic representation for image Segmentation steps

\section{B. Feature Extraction}

Segmentation operated and reduced pixels number of the pigmented lesion to save time dealing just with the required region. Then sets of different features were extracted to represent the lesion essential characteristics for accurate discrimination between benign and malignant lesions. The most valuable prognostic factor of malignant melanoma is appearance variability. It is irregular in shape and color with differential structure.

On this spot, that research attempt to compromise that heterogeneous phenomena proposing and examining huge different number of features. The features presented study lesion property from three concerned sides geometric for shape, chromatic for color and texture features representing differential structure. Texture features were studied in details in regard to different color spaces and coordinates with respect to GLCM. This section will discuss the different types of features used through this study to correctly define the PSL introduced.

a) Geometric Features: Shape descriptors supply important information to discriminate malignant melanoma from melanocytic nevi. Results shows that geometric features are usually efficient in analayzing region of interest shape in a binary image. This study computed 21 geometric features [9, 32] to describe a set of properties for the segmented binary image of PSL. Fig. 6 represents a schematic representation of the binary image that shows the geometric represenation of each parameter calculated considered as a separate feature as follow. Area $\left(\mathrm{A}_{\mathrm{p}}\right)$, Perimeter $\left(\mathrm{P}_{\mathrm{p}}\right)$, Center of gravity (Centroid), Solidity $\left(A_{p} / A_{c}\right)$, Roundness, Irregularity, Major axis $\left(a_{p}\right)$, Minor axis $\left(b_{p}\right)$, Ratio of axis $\left(a_{p} / b_{p}\right)$, Eccentricity, Extent $\left(A_{p} / A_{b}\right)$, Equivalent Diameter, Radius, Bounding Box area $\left(A_{b}\right)$, $x$-axis of bounding box $\left(b_{b}\right), y$-axis of bounding box $\left(a_{b}\right)$, Ratio of bounding box axis $\left(b_{b} / a_{b}\right)$, Estimated borderline, Convex Hull, Convex Hull area (Ac), Orientation.

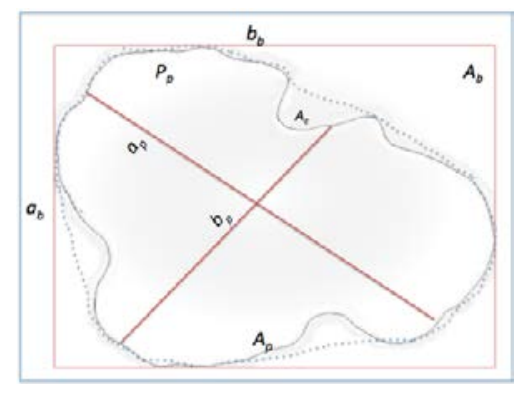

Fig. 6. Binary image lesion representation

b) Chromatic Features: Geometric features were promising in describing the lesion physical structure, but it missed the signifigance of tumor color, brightness or luminance that could be aguide to distinguish PSL true specification. Different previouse studies [33] had proven that color features is useful and robust cue for diagnosing malignant melanoma. Skin lesion could be examined by different color spaces rather than RGB. Variouse color spaces was introduced through imaging processing field. Color space could be simply defined as different mathematical representation for a set of colors in ways that make certain calculations more convenient, where they provide a method to identify colors that is more intuitive. This section deals brefily with the seven color spaces introduced through this study and the different features vectors extracted from each.

The seven color spaces introduced to study the PSL through them are RGB, HSV,HIS,YIQ, YCbCr, L*u*v* and lab spaces[33-35]. The most common space is the true color space represented by the RGB space, it defines a color as the percentage of red, green and blue hues colors mixed. Also the intuitive notions of hue, saturation, and brightness was represnted by HSI and HSV color spaces to simplify processing and identify more details and information about the lesion [34]. In the field of video standards, the most famouse color spaces are YIQ and YCbCr. The Y component represents the luminance information, it is the only component used by black-and-white television receivers where $\mathrm{I}, \mathrm{C}_{\mathrm{b}}$ and $\mathrm{Q}, \mathrm{C}_{\mathrm{r}}$ represent the chrominance information [33]. However; the five different color spaces mentioned above can help in studing the PSLs but it was interesting to pursuit other valuable color spaces, such as $\mathrm{L}^{*} \mathrm{u}^{*} \mathrm{v}^{*}$ and lab spaces [35]. According to the breifly defined color spaces, this study will investigate statistical features depending on each color space. The three different statistical features extracted from each component of the color spaces are specifically the mean, standard deviation, 'coefficient of variation' and the sum of color space component. 
1) Mean $(\mu)$ : It is calculated as the average of the intensity pixels belonging to the lesion.

2) Standard Deviation ( $\sigma)$ : This value was taken as the color variation risk. In general, lesions with a higher color standard deviation are considered to be a higher cancer risk. This feature can be represented by the satndard deviation value for all the pixels belonging to lesion regions with respect to the 7 different color spaces previously disscussed.

3) Coefficient of variation: It can be seen as the relative standard deviation that can be represented by division of the leasion standard deviation by its mean value.

4) Sum of color component: Another new chromatic feature was proposed that considered the sum of the three channels intensity values of the color space, i.e for the YIQ space the feature represented by the sum of the channel value for luminace $(\mathrm{Y})$, and the 2 chromatic components (I and Q), as $\mathrm{S}=\mathrm{Y}+\mathrm{I}+\mathrm{Q}$

Regarding the seven different color spaces mentioned through the above paragraphs and the four parameters explained, 10 features extracted from each space. These results the 70 different chromatic features presented through this study. Fig.7. shows an example for malignant melanoma region represented by the 7 different color spaces to show the different effect of each upon the PSL.

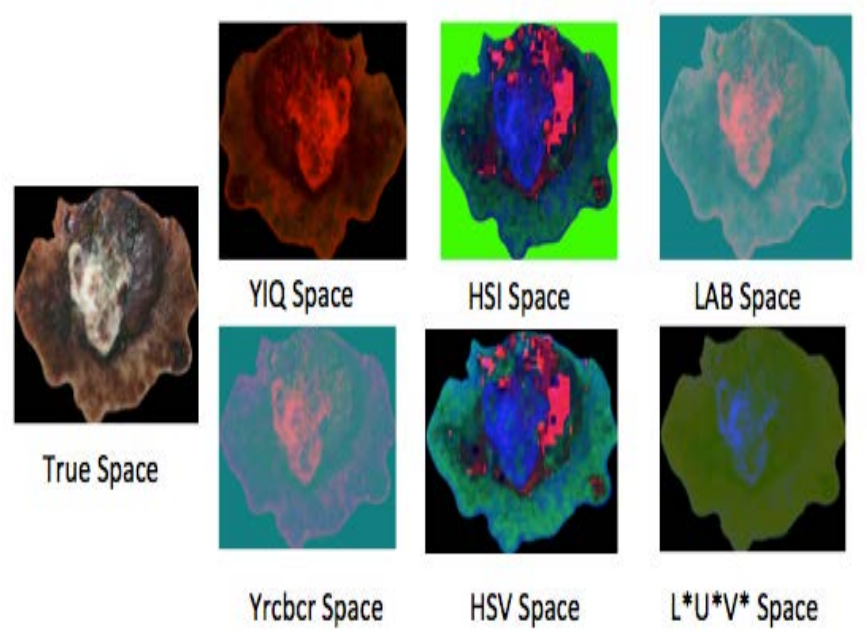

Fig. 7. Malignant melanoma lesion representation regarding the 7 color spaces

c) Texture Features: Skin lesion physical shape and color was essentially considered through morphological features. In more advanced melanoma, the skin on the surface may break down and look scraped. Also it may become hard or lumpy; the surface may ooze or bleed. Textures represent tonal variations in the spatial domain and determine the overall visual smoothness or coarseness of image features. Accordingly; texture features extractions stand as the essential accomplishment step to have complete and efficient approach. Texture features are considered to be one of the widely used tools in malignant tissue detection in digital images, where it can describe melanoma's different structures. Texture processing algorithms are usually divided into three major categories: structural, spectral and statistical [36,37].
Statistical texture features have been proven to be powerful in classifying malignant and benign tissues. Texture analysis covers a wide range of techniques based on first- and second order image texture parameters, as the first order statistical features are not at all sufficient to characterize the heterogeneity of the different tumor types.

Since different lightening conditions change the apparent texture of a lesion [38], in addition to these two different groups of texture features it was also interesting to discuss tumor texture with respect to the 7 different color spaces previously proposed to circumvent lightening problem and coup with any variation. Toward the main aim of discrimination between normal and abnormal lesion, this section will deal with all texture features extracted starts with first order statistical features, higher order statistical features (GLCM) and then texture features of 7 color spaces.

1) First Order Statistical features: First order statistics can be used as the most basic texture features and are properly named just statistical rather than texture feature. Two groups of statistical features were proposed; one group based on the probability of pixel's intensity values occurring in digital images and independent on spatial distribution of pixels, the other group depends on the histogram statistics. The first order features were extracted depending on spatial distribution of pixels was Mean, Standard deviation, Smoothness, Third Moment and Entropy. Histogram features are extracted depending on the calculated histogram of the lesion image; the six features extracted based on histogram are Mean, Skewness, Entropy, kurtosis, Energy and variance.

2) Higher order statistical features: They are second order features that depend on pixel value and their spatial inter-relationships. Higher or second order statistical features are based on statistical parameters such as the Spatial Gray Level Dependence Method (co-occurrence matrices), the Gray Level Difference Method, and the Gray Level Run Length Matrices $[39,40]$. One of those examples proposed for that study is the gray level co-occurrence matrix, as it is most popular texture analysis used previously for discrimination of melanoma [9,41]. The choice of Haralick features [42,43] based on GCMs was made considering their proven applicability to analyze objects with irregular outlines [44].

Gray level co-occurrence matrix (GLCM) is a tabulation of how often different combinations of pixel brightness values (grey levels) occur in an image. It is constructed by observing pairs of image cells separated distance $d$ from each other and incrementing the matrix position corresponding to the grey level of both cells. Its operation can be simply discussed through the schematic representation example shown on Fig.8 The 21 higher order texture features [42] used in this study are autocorrelation, contrast, correlation, cluster shade, cluster prominence, dissimilarity, energy, entropy, homogeneity, maximum probability, sum of squares (Variance), sum of average, sum of variance, sum of entropy, difference variance, difference entropy, Information measure of correlation 1, Information measure of correlation2, Inverse difference (INV) is homom, Inverse difference normalized (INN), Inverse difference moment normalized (IDM). 


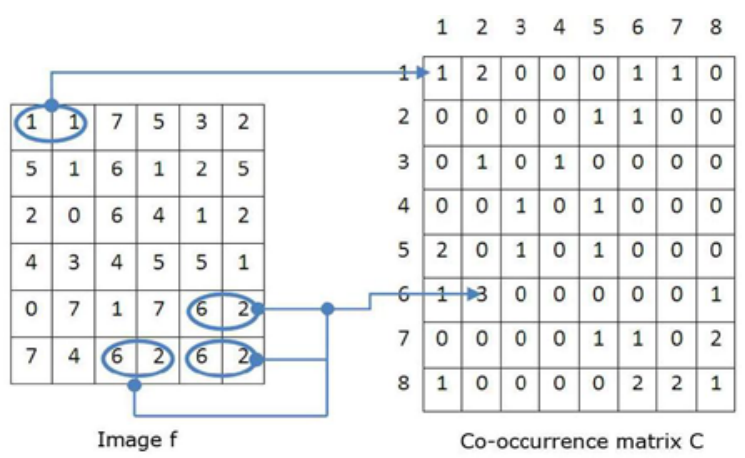

Fig. 8. Example of gray level co-occurrence matrix (GLCM)

The GLCM is determined using gray-tone spatialdependence matrices $D_{s}^{[K]}(i, j)(K=1, \ldots, 4)$ listening how many times the gray tones $i$ and $j$ are spatial neighbors. So, various co-occurrence matrix realizations depending on distance and angle, and displaying different angular relationships, are introduced through our wok. The textural measures were calculated on horizontal, vertical and two diagonal directions on an image as $D_{s}^{[K]}(i, j)$ is computed for each of the four angles $0^{\circ}, 45^{\circ}, 90^{\circ}$ and $135^{\circ}$, where $K$ denotes the angle of spatial neighborhood. Fig.9 illustrates the geometrical relationships of GLCM measurements made by the four angles under the assumption of angular symmetry. The previously mentioned 21 GLCM features extracted were defined in the four different angles (21 features *4 different angles) to have for total 84 features [45].

$135^{\circ}[\cdot D \cdot D]$

$90^{\circ}[\cdot 00]$

$45^{*}[\cdot-00]$

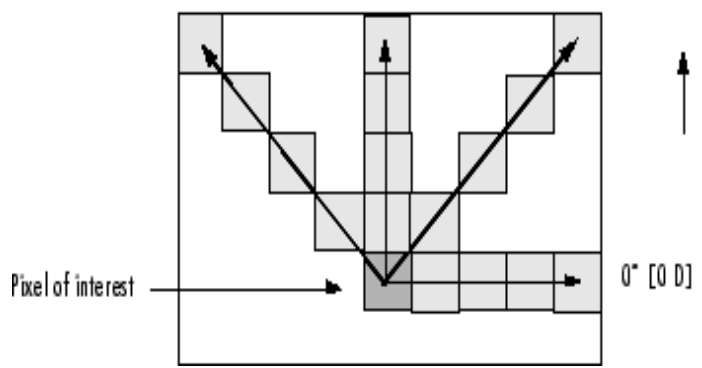

Fig. 9. Geometry directions of adjacency for calculating GLCM features

Texture features of 7 color spaces: Important features about skin texture were extracted by evaluating some statistical parameters about the PSL in regard to the different 7 color spaces mentioned in the chromatic section. In regard to the powerful indication of the tumor specification resulted from measuring its entropy, it was powerful to measure entropy for the PSL based on the 7 color spaces to result 7 different texture features. Furthermore, texture analysis includes several functions that filter an image using standard statistical measures such as range, standard deviation [46]. These statistics can characterize the texture of an image because they provide information about the local variability of the intensity values of pixels in an image. For example, in areas with smooth texture, the range of values in the neighborhood around a pixel will be a small value; in areas of rough texture, the range will be larger. Accordingly, the means and standard deviations of local entropy [46], local standard deviation and local range [47] of gray scale image are added as separate features for each three color component of the seven spaces $(2 \times 3 \times 7 \times 3)$ counting out one hundred and twenty- six feature.

Further information about skin texture can be mentioned by extracting GLCM features out of image transformed to the different color spaces used. Based on the co-occurrence matrix, many different texture descriptors may be computed. To reduce the computational complexity, only four most relevant features were selected out of 21 GLCM features presented above, namely Energy, Homogeneity, Correlation and Contrast based on their classification accuracy, computational cost and previously usage in literature [9]. About 88 features were extracted through the present section, where each of the four features was extracted out of each color component of the 7 color spaces $(7 \times 3 \times 4)$, In addition to the four features extracted from the gray scale image of the true space on RGB color.

In conclusion, this study presented huge numbers of texture features to fulfill the aim toward having complete definition for the PSL structure and specification. Three hundred-sixteen texture features were extracted considering eleven first order statistical features, eighty-four second order statistical features in addition to texture representation regarding the different seven color spaces, which presented two hundred and twenty one feature. A Simple representation of the distribution for all texture features extracted based on different color spaces is shown in Fig.10.

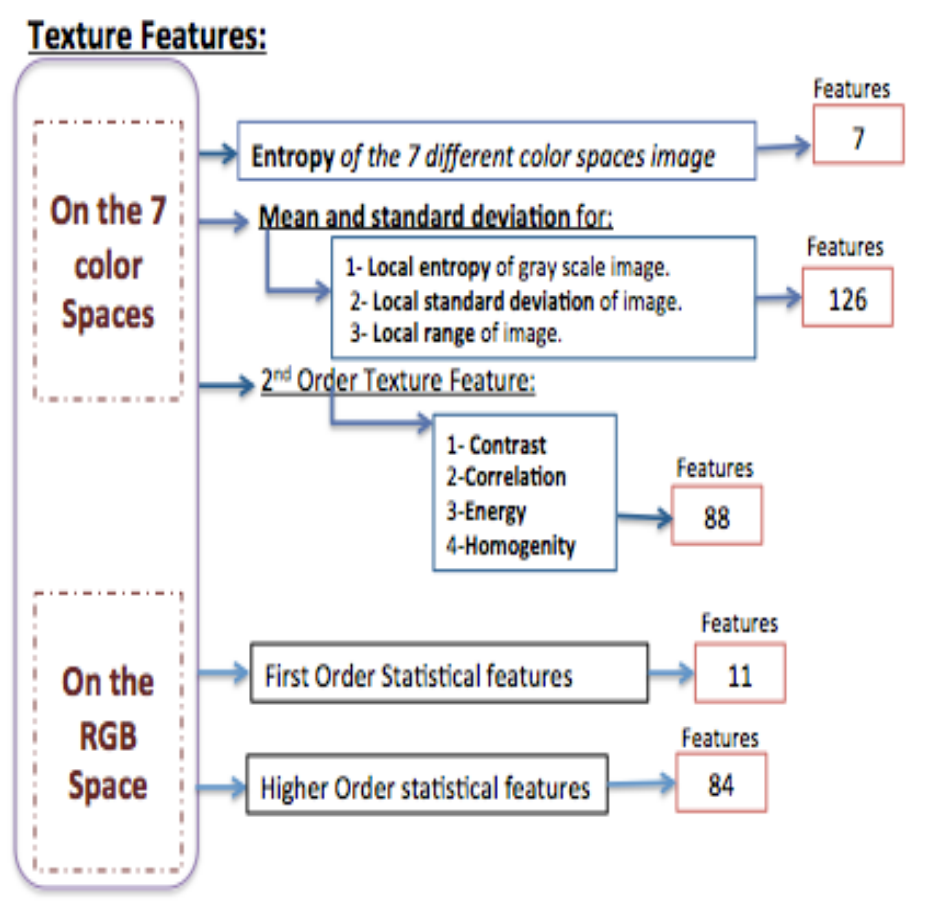

Fig. 10. Texture features diagram

\section{Feature Selection}

Although, features variety are helpful to screen out tumor properties for accurate diagnosis, but approaches have this huge number of features usually suffer complexity. It is 
difficult to experimentally analyze and explicitly recognize patterns in the data of a larger test set, where it will in turn increase processing time, as some of these features are depend on heavy calculations. Features are not equally efficient for classification task due to redundancy or irrelevance. Thus, feature selection techniques used to reduce features quantity significantly, while increasing the detection accuracy. The main idea of feature selection is to choose a subset of input features by eliminating features with little or no predictive information.

All methods begin by having all samples in the feature space of unknown class assignment. The methods described through this study will assign classes by measuring how nearer the samples to a certain limit and similar samples will be grouped by assigning them the same class. Three attribute selection algorithms are used for the dimensionality reduction and identification of the most prominent features to capture efficiently the system. The three statistical methods proposed for the target of feature selection was, the F-test $[48,49]$, the T-test [50] and the Fisher score ranking [51] to compare and increase the detection accuracy.

In statistical significant testing T-test and F-test, the pvalue is the significant level of probability. The smaller the pvalue, the more significant is the difference between the distributions of the two groups. Features with practically different variance was chosen as significant features where their p-values are suppose to be less than the significant level used for that study which is 0.05. Fig.11 shows simple description for t-value, which used to measure the correlation regarding $\mathrm{P}$-value in the t-test selection method. On the contrary, features selected according to fisher score ranking are those with the highest ranks, where the ranking threshold for this study was 0.5 .

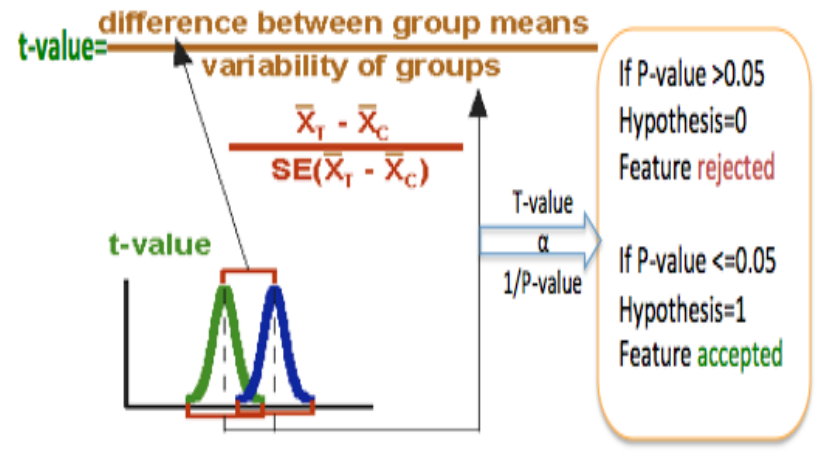

Fig. 11. T-test and P-value relation

\section{Classification}

The final goal of a skin cancer automatic detection system is to build a decision rule that will discriminate between malignant and benign lesions. Automatic diagnosis system is usually accomplished by means of the classification process, that define as a finding operation for a set of attributes to distinguish between classes of data for the purpose of predicting the class of objects, whose class label is unknown. Generally a classifier generates a decision boundary, which separates the points in feature space into two or more sets of classes. Each type of classifier will arrive at a decision boundary in a different way according to its working process and the classifier types. Toward accurate diagnosis for PSL different classifying algorithms introduced based on Artificial Neural Network (ANN), the K-Nearest Neighbor classifier (KNN) and Support Vector Machine (SVM).

This section concerned with discussing the main idea of the three different classification models proposed to differentiate between malignant and benign lesions. As mentioned through material section this study introduces 160 images for each data set whether dermoscopic or clinical image. All images are fairly divided for two classes $50 \%$ for learning set and 50\% for test set. So, 80 images representing 40 for malignant melanoma cases and 40 of benign nevi cases used as a training set to accomplish the automated detection system of tumors, whereas the other 80 image having the same distribution for testing the automated detection system.

a) Artificial Neural Network (ANN): Artificial neural networks are computational paradigms based on mathematical models that unlike traditional computing have a structure and operation, which resembles the mammal brain. Artificial neural networks or neural networks for short, are also called connectionist systems, parallel distributed systems or adaptive systems, because they are composed by a series of interconnected processing elements that operate in parallel. The main idea of computer-aided diagnosis was to design software having nearly functionality of the human eyes to extract features and the brain to classify them. Thus, ANN was the first choice of classification algorithm among all CAD software programmers [52].

Generally, ANN Classification process usually operated through two successive steps; learning (or training) and testing. The training phase learns the set of examples presented to the network, where the network 'guesses' the output for each example. As the training goes on, network internally adjust until it reaches a stable stage at which the provided outputs are satisfactory. Neural networks can learn in two ways: supervised or unsupervised [52].

To decide on a network topology some points must be considered for designing ANN classifier, Number of input nodes represented by the number of selected features feeded into the classifier. Number of output nodes is just one where it suppose to have a clear-cut if that image is whether malignant or benign. Then, Number of hidden nodes and Activation function are selectable according to the designed network and finally the network Training behavior. Accordingly two techniques were proposed for our study, the Automatic and Traditional ANN technique [12].

b) K-Nearest Neighbor (KNN): KNN has recently been recognized as one of the best algorithms for clustering data as it is simple, quick and effective. $\mathrm{K}$ indicates the number of neighbors, then each sample, is classified in the same class, as the training data is closest [53]. In other words, there is no explicit training phase or it is very minimal and pretty fast. Each classified sample neighbour in KNN training phase is considered as an item of evidence which supports certain hypotheses regarding the class membership of that pattern. 
Finally the test image is classified as belonging to the class, which is most frequent among the K-samples nearest to it.

Support Vector Machine (SVM): Vapnik and Lerner are the first investigators who developed the Support Vector Machine (SVM) algorithm in 1963. The theory has been improved to generalize the ability of learning machines regarding unseen data [54,55]. In the last few years SVM have shown perfect performance in different real-world applications including object recognition and image face detection [56-59]. SVM is based on the idea of minimizing the generalization error when the classifier is applied to test samples, by estimating its magical function that can powerfully classifies data into two classes. Finding the hyper plane that can perfectly discriminate between all data points is the main idea of SVM algorithm. The best hyper-plane for the SVM means the one with the largest margin between the two classes, where margin is the maximal width of the slab parallel to the hyper-plane that has no interior data points. Margin is usually maximized when the norm of the weight vector is minimum. Fig. 12 illustrates SVM methodology of classification.

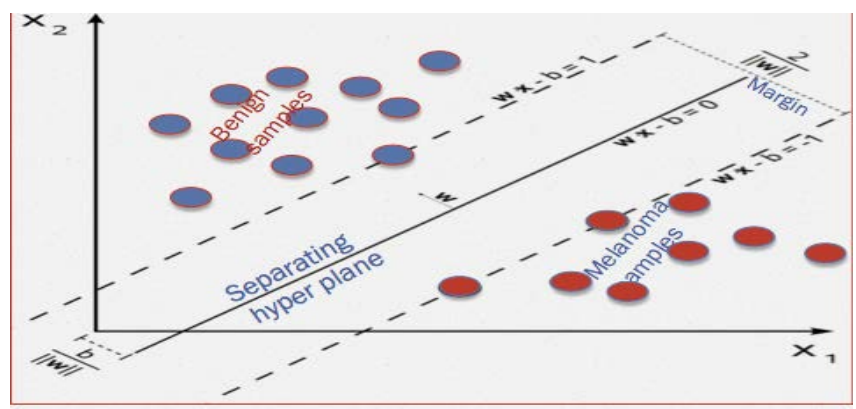

Fig. 12. SVM Classification

In order to obtain satisfactory predictive accuracy, different SVM kernel functions were proposed. Regarding the difference occurred between classifiers due to their training procedure. Three different SVM approaches have been proposed for classification as a result of using three different kernel functions that are linear, quadratic and RBF kernels [60-62].

\section{E. Performance measure}

The previously mentioned algorithms and models for detecting melanoma rely on the automatic assessment of the skin to interpret whether a pigmented skin lesion is a melanoma or not. All software developed will have an error rate and occasionally will either fail to identify an abnormality, or well identify an abnormality that is not present. To be able to evaluate the results and assign some kind of performance measure to the diagnosis done by the system. Statistical measures called sensitivity and specificity calculated to evaluate the diagnostic accuracy, calculated through equation the following three equations 3,4 and 5 . These measures are used to describe the error rate by the terms true and false positive and true and false negative $[63,64]$.

$$
\begin{aligned}
& \text { Sensitivity }=\mathrm{TP} /(\mathrm{TP}+\mathrm{FN}) * 100 \%(3) . \\
& \text { Specificity }=\mathrm{TN} /(\mathrm{TN}+\mathrm{FP}) * 100 \%(4) . \\
& \text { Accuracy }=(\mathrm{TP}+\mathrm{TN}) /(\mathrm{TP}+\mathrm{TN}+\mathrm{FP}+\mathrm{FN}) * 100 \%(5) .
\end{aligned}
$$

\section{EXPERIMENTAL RESULTS}

The previous methodology section illustrated the steps performed for the purpose of PSL diagnosis. As all database images processed and segmented then features were extracted and the most significant was selected. Finally, the promoted features worked as input for the three mentioned classifiers. As result of the huge number of features extracted and the different methods used in both feature selection and classification process, there will be multi different experimental results. This section intent to monitor the classification results according to the testing phase, then, analyzes its performance among different features used. Measuring performance to such huge number of experiments was handled by dividing experiments according to features category. Experimental approaches were divided into single and combined models; Fig. 13 shows the description of the different approaches applied for both single and combined models.

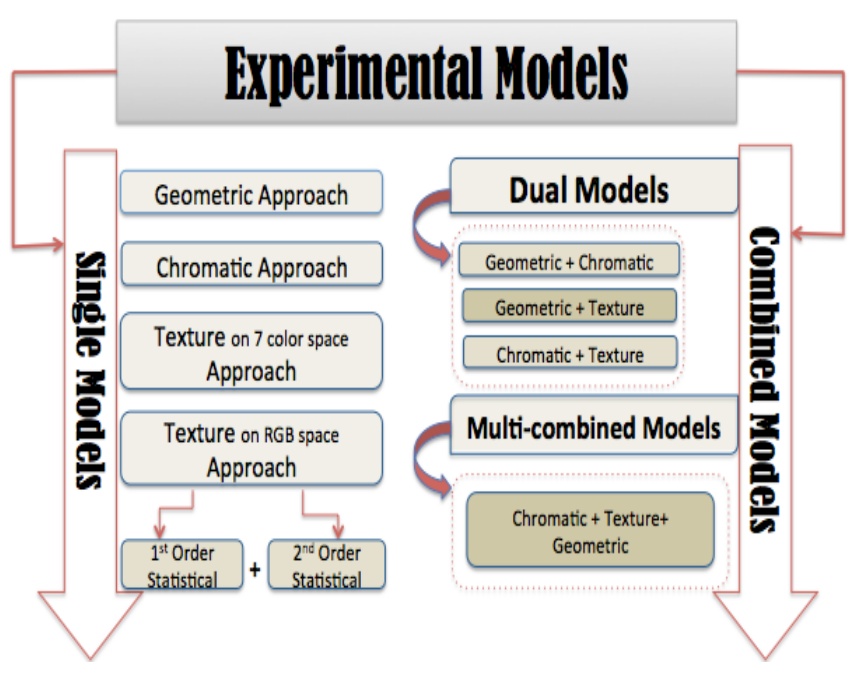

Fig. 13. Single and Combined Model approaches

\section{A. Single Models}

These models focus on studying a single type of feature and compare its performance when using three feature 
selection methods to choose the most significant features that act as input to different classifiers. According to the different types of features extracted, four single models are proposed. Regarding the 3 sets of chosen features, resulted from f-test, ttest and fisher score raking, fed into the 6 different classification processes applied on both dermoscopic and clinical data set, 36 different experiment is resulted from each single approach. Fig. 14. Shows the general schematic representation for single approach.

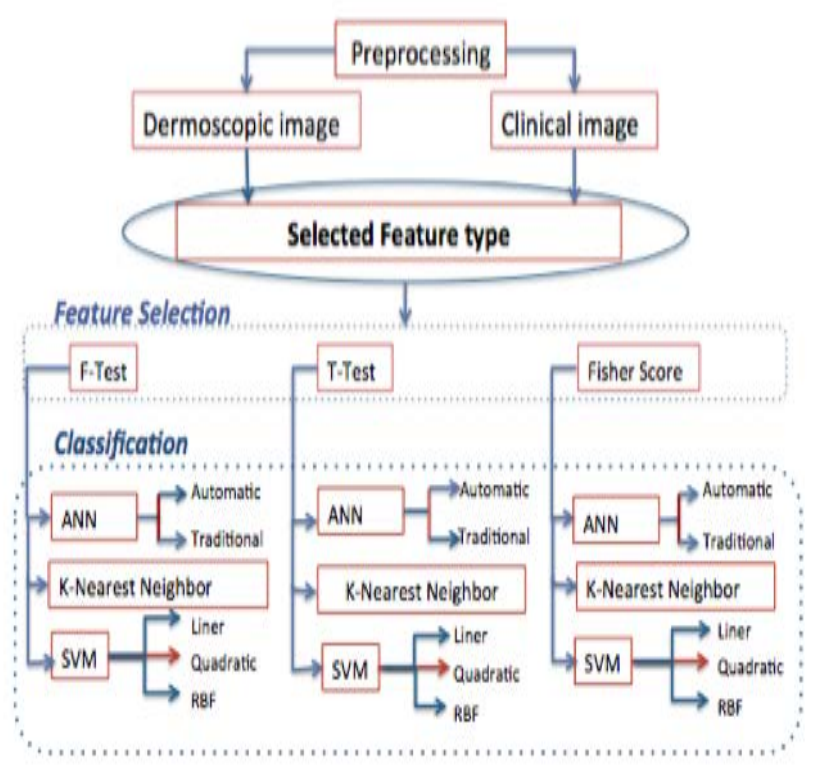

Fig. 14. Single Model Block Diagram

The best accuracy recorded from of all single approach experiments' carried out is discussed as follow:

a) Geometric Approach: The Fisher score method defines the most significant feature for both image types among the geometric features. The most accurate model was presented by introducing the 15 geometric features selected by fisher score ranking to SVM and ANN, which recorded 93.7\% regarding both dermoscopic and clinical database.

b) Chromatic Approach: The best two models performed by chromatic features regarding both dermoscopic and clinical images were classified using ANN. Results regarding both datasets were promising where models recorded $92.5 \%$ using t-test selected features and $93.75 \%$ using fisher selected features for and dermoscopic and clinical database respectively.

c) Texture of 7-color space Approach: Texture features were not very promising for digital images rather than dermoscopic lesions. Clinical data reaches accuracy of $87.5 \%$ using t-test features when classified by ANN. On the other hand, Dermoscopic data reach $90 \%$ when fisher-selected features were classified by linear SVM.

d) Texture Analysis using the RGB space Approach: Through this feature category, the best accuracy recorded for both database types was 87.5 regarding different models. The best dermoscopic model was built up using features selected by f-test model and classified with linear and quadratic SVM. For clinical database, it was observed that the best accuracy recorded was $87.5 \%$ according to feature selected by t-test and classified by traditional ANN.

According to the previous results it was observed the first three single approaches (geometric, chromatic and texture based on 7 color spaces) were much more promising than texture features based on RGB space. Thus, first and second order statistical features based only on RGB space were excluded from the group of features used to construct combined models.

Regarding the three selected features category selected it was observed that feature selected upon using the T-test and Fisher score method shows the best results. But since the t-test usually selects a larger set of features compared to the fisher score method reaching very relative results, fisher score method was preferred. Accordingly, the features selected by the fisher score used for the combined model as the most significant. It is valuable to determine the most significant features could be used to determine melanoma, table 1 mentions all the selected features according to the three different categories (Chromatic, Geometric and Texture).

Fifteen and twenty-two features represent geometric and chromatic features respectively for both the dermoscopic and clinical database. Forty- seven and twenty texture features selected to represent dermoscopic and clinical database respectively.

\section{B. Combined Models}

After studying each type of features separately through the single models, it was valuable to monitor the effect of combining the most revealing features of each type. Four combined approaches were proposed as a result of amalgamation between the best features extracted out of each feature type. Three approaches were considered by combining two different feature types together (Geometric + Chromatic), (Chromatic + Texture) and (Geometric + Texture), dual models could name those. The fourth approach was proposed by combining the three feature types together (Geometric + Chromatic + Texture).

Therefore, combined models study different types of features together and compare their performance when best features cooperate and feed as input for the different applied classification algorithms. Fig. 15, easily mention the process that the combined model goes through. 


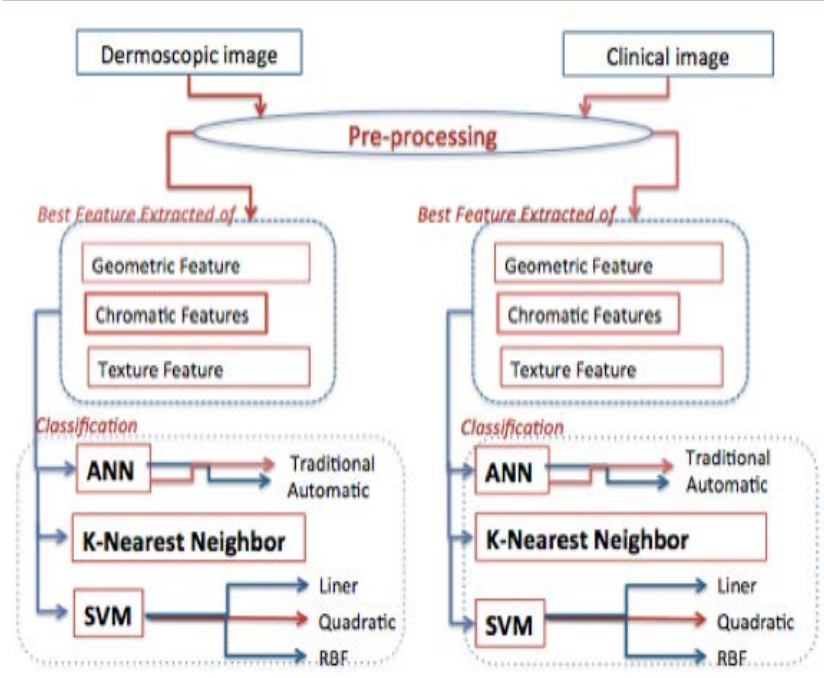

Fig. 15. The Combined Model Block diagram

a) Dual Models: Geometric features record the highest results when combined with texture or chromatic features for the two different types of databases. The best accuracy recorded was $95 \%$ for geometric and chromatic features sets of dermoscopic images classified by linear SVM. Where also the combination between geometric and texture feature of clinical images recod the same percentage when classified by automated ANN.

1) Dermoscopic database: A number of thirty-seven, sixty-two and sixty-nine features were considered the total significant features composed for the three approaches (Geometric + chromatic), (Geometric + Texture) and (Chromatic+ texture), respectively. It was observed that best accuracy recorded was $95 \%$ for the combination of geometric and chromatic features classified by linear SVM.

2) Clinical database: A number of thirty-seven, thirtyfive and forty-four features were considered the total significant features composed of the three approaches (Geometric + chromatic), (Geometric+ Texture) and (Chromatic+ texture), respectively. It was observed that best accuracy recorded was $95 \%$ for the combination of geometric and texture features classified by automated ANN.

a) Multiple Models: It was further observed that the amalgamation between the three different features category concluded a well representation for lesions whether of dermoscopic or clinical images.

1) Dermoscopic database: Fifteen, twenty-two and fortyseven geometric, chromatic, and texture features respectively were introduced to the six different classification methods to evaluate the model performance. It was remarked that the most accurate model was reached by classifying the three combined features according to the traditional mode of ANN, that record accuracy of $96.25 \%$. Also it was most remarkable that linear SVM yielded very good results that reach $100 \%$ for sensitivity and record accuracy 95\%.

2) Clinical database: Fifteen, twenty-two, and twenty geometric, chromatic and texture features respectively, were introduced to the six different classification methods to evaluate the model performance. The most accurate model performed to diagnose the PSL was represented in clinical images by classifying the three combined features according to traditional mode of ANN that recorded 97.5\%. This was followed by the automatic validation mode of ANN with a recorded accuracy of $95 \%$.

\section{Dermoscopic Combined Models}

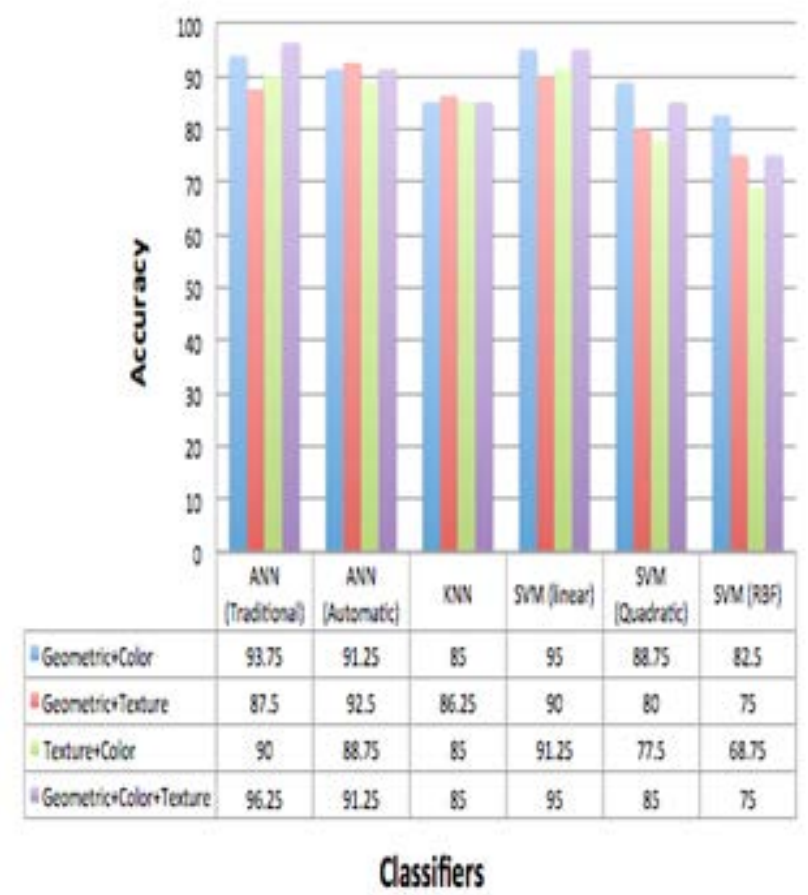

Fig. 16. Dermoscopic combined models results

\section{Clinical Combined Models}

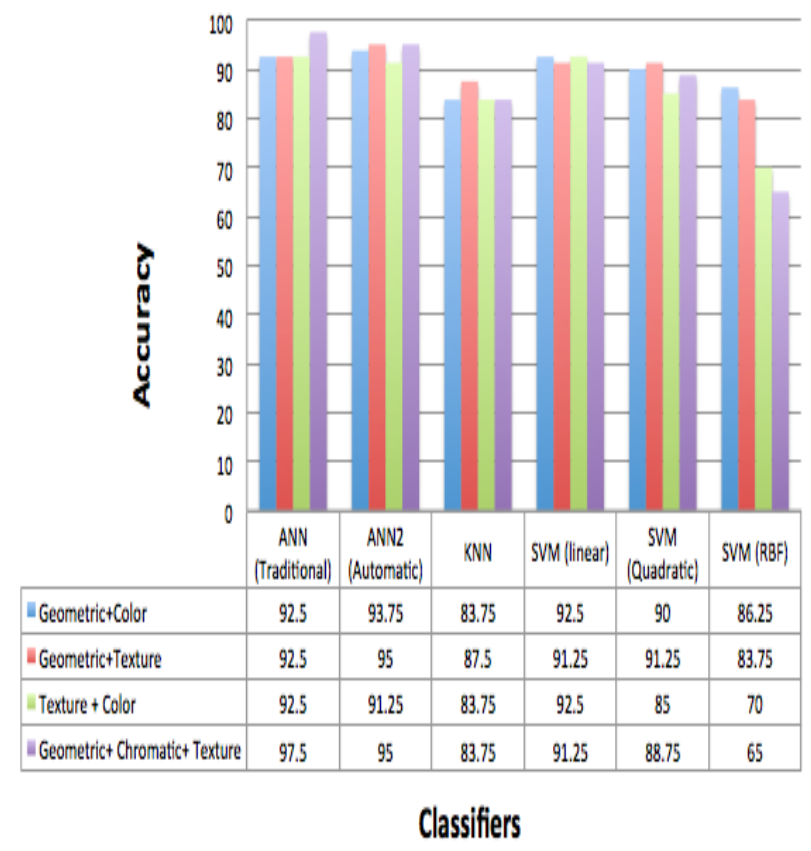

Fig. 17. Clinical combined models results 
(IJACSA) International Journal of Advanced Computer Science and Applications,

TABLE I. The Selected Features According to Fisher Score Method

\begin{tabular}{|c|c|c|c|c|}
\hline \multicolumn{5}{|c|}{ Selected features } \\
\hline Geometric & \multicolumn{2}{|c|}{ Chromatic } & \multicolumn{2}{|c|}{ Texture } \\
\hline $\begin{array}{l}\text { Dermoscopic \& } \\
\text { Clinical Features }\end{array}$ & $\begin{array}{l}\text { Dermoscopic } \\
\text { Features }\end{array}$ & Clinical Features & Dermoscopic Features & Clinical Features \\
\hline Equivalent Radius & Mean of Red (RGB) & Mean of H (HIS) & Entropy of RGB & $\begin{array}{l}\text { Standard deviation of Local entropy for } \\
\text { R, G \& B of (RGB). }\end{array}$ \\
\hline Area & Mean of Y (YIQ) & Mean of I of (YIQ) & $\begin{array}{l}\text { Mean of Local entropy for G and B } \\
\text { (RGB) }\end{array}$ & $\begin{array}{l}\text { Standard deviation of Local entropy for } \\
\text { H \& I of (HSI). }\end{array}$ \\
\hline Perimeter & Mean of V (HSV) & Mean of S of (HSV) & $\begin{array}{l}\text { Mean of Local standard deviation for } \\
\mathrm{u}^{*} \text { and } \mathrm{v}^{*}\left(\mathrm{~L}^{*} \mathrm{u}^{*} \mathrm{v}^{*}\right)\end{array}$ & $\begin{array}{l}\text { Standard deviation of Local entropy for } \\
\text { Y of (YIQ). }\end{array}$ \\
\hline Solidity & Mean of Y (Ycbcr) & Mean of cr of (Ycbcr) & Mean of Local range for $\mathrm{u}^{*}\left(\mathrm{~L}^{*} \mathrm{u}^{*} \mathrm{v}^{*}\right)$ & $\begin{array}{l}\text { Standard deviation of Local entropy for } \\
\text { H, S \& V of (HSV). }\end{array}$ \\
\hline Roundness & Mean of L (Lab) & Mean of a (Lab) & $\begin{array}{l}\text { Standard deviation of Local entropy } \\
\text { for S and V of (HSV) }\end{array}$ & $\begin{array}{l}\text { Standard deviation of Local entropy for } \\
\text { Y of (Ycbcr). }\end{array}$ \\
\hline Extent & Mean of $\mathrm{u}^{*}\left(\mathrm{~L}^{*} \mathrm{u}^{*} \mathrm{v}^{*}\right)$ & Mean of cb (Ycbcr), & $\begin{array}{l}\text { Standard deviation of Local standard } \\
\text { deviation for } R, G \& B\end{array}$ & $\begin{array}{l}\text { Standard deviation of Local entropy for } \\
\text { L of (Lab). }\end{array}$ \\
\hline Irregularity & $\begin{array}{l}\text { Standard deviation } \\
\text { of } \mathrm{u}^{*}\left(\mathrm{~L}^{*} \mathrm{u}^{*} \mathrm{v}^{*}\right)\end{array}$ & Mean of b (Lab) & $\begin{array}{l}\text { Standard deviation of Local standard } \\
\text { deviation for S and I of (HSI) }\end{array}$ & $\begin{array}{l}\text { Standard deviation of Local entropy for } \\
\mathrm{L} \& \mathrm{u}^{*} \text { of }\left(\mathrm{L}^{*} \mathrm{u}^{*} \mathrm{v}^{*}\right) \text {. }\end{array}$ \\
\hline Major Axis & Sum of $(R+G+B)$ & Mean of $\mathrm{L}\left(\mathrm{L}^{*} \mathrm{u}^{*} \mathrm{v}^{*}\right)$ & $\begin{array}{l}\text { Standard deviation of Local standard } \\
\text { deviation for Y of (YIQ) }\end{array}$ & $\begin{array}{l}\text { Standard deviation of Local Std for } \mathrm{u}^{*} \\
\text { of }\left(\mathrm{L}^{*} \mathrm{u}^{*} \mathrm{v}^{*}\right) \text {. }\end{array}$ \\
\hline Minor Axis & Sum of $(L+a+b)$ & Mean of $\mathrm{u}^{*}$ of $\left(\mathrm{Lu}^{*} \mathrm{v}^{*}\right)$ & $\begin{array}{l}\text { Standard deviation of Local standard } \\
\text { deviation for V of (HSV) }\end{array}$ & $\begin{array}{l}\text { Standard deviation of Local range of } \\
\text { image for } \mathrm{u}^{*} \text { of }\left(\mathrm{L}^{*} \mathrm{u}^{*} \mathrm{v}^{*}\right) \text {. }\end{array}$ \\
\hline Equivalent Diameter & Sum of $(H+S+I)$ & $\begin{array}{l}\text { Standard deviation of S } \\
\text { (HSV). }\end{array}$ & $\begin{array}{l}\text { Standard deviation of Local standard } \\
\text { deviation for Y of (Ycbcr) }\end{array}$ & GLCM Energy of H of (HSI). \\
\hline Bounding box area & Sum of $\left(L+u^{*}+v^{*}\right)$ & Sum of: $(\mathrm{R}+\mathrm{G}+\mathrm{B})$ & $\begin{array}{l}\text { Standard deviation of Local standard } \\
\text { deviation for } \mathrm{L} \text { of (Lab) }\end{array}$ & GLCM Energy of H of (HSV). \\
\hline $\mathrm{X}$-axis of bounding box & Sum of $(\mathrm{Y}+\mathrm{I}+\mathrm{Q})$ & Sum of $(\mathrm{H}+\mathrm{S}+\mathrm{I})$ & $\begin{array}{l}\text { Standard deviation of Local std for } L \\
\& u^{*} \& v^{*} \text { of }\left(L^{*} u^{*} v^{*}\right)\end{array}$ & GLCM Energy of cr of (Ycbcr). \\
\hline Y-axis of bounding box & Sum of $(H+S+V)$ & Sum of $(\mathrm{Y}+\mathrm{I}+\mathrm{Q})$ & $\begin{array}{l}\text { Standard deviation of Local range for } \\
\text { R and G (RGB) }\end{array}$ & GLCM Energy of (Ycbcr). \\
\hline Estimated border line & $\begin{array}{l}\text { Coefficient variant } \\
\text { Of Red }\end{array}$ & Sum of $(H+S+V)$ & $\begin{array}{l}\text { Standard deviation of Local range for } \\
\text { I (HSI), }\end{array}$ & GLCM Energy of b of (Lab). \\
\hline \multirow[t]{15}{*}{ Convex hull area } & $\begin{array}{l}\text { Coefficient variant } \\
\text { Of I (HIS) }\end{array}$ & Sum of $(L+a+b)$ & $\begin{array}{l}\text { Standard deviation of Local range for } \\
\text { Y (YIQ) }\end{array}$ & GLCM Energy of $\mathrm{u}^{*}$ of $\left(\mathrm{Lu}^{*} \mathrm{v}^{*}\right)$. \\
\hline & $\begin{array}{l}\text { Coefficient variant } \\
\text { Of Y (YIQ) }\end{array}$ & Sum of $\left(L+u^{*}+v^{*}\right)$ & $\begin{array}{l}\text { Standard deviation of Local range for } \\
\text { V (HSV) }\end{array}$ & \\
\hline & $\begin{array}{l}\text { Coefficient variant } \\
\text { Of S (HSV) }\end{array}$ & $\begin{array}{l}\text { Coefficient variant Of } \\
\text { Red }\end{array}$ & $\begin{array}{l}\square \text { Standard deviation of Local range } \\
\text { for } \mathrm{L}, \mathrm{u}^{*}, \mathrm{v}^{*}\end{array}$ & \\
\hline & $\begin{array}{l}\text { Coefficient variant } \\
\text { Of V (HSV) }\end{array}$ & $\begin{array}{l}\text { Coefficient variant Of S } \\
\text { (HSV) }\end{array}$ & $\begin{array}{l}\text { Standard deviation of Local range for } \\
\text { Y (Ycbcr), }\left(L^{*} u^{*} v^{*}\right) \text {, }\end{array}$ & \\
\hline & $\begin{array}{l}\text { Coefficient variant } \\
\text { Of Y (Ycbcr) }\end{array}$ & $\begin{array}{l}\square \text { Coefficient variant Of } \\
\text { V (HSV) }\end{array}$ & GLCM Homogeneity of RGB, R, G & \\
\hline & $\begin{array}{l}\text { Coefficient variant } \\
\text { Of L (Lab) }\end{array}$ & $\begin{array}{l}\text { Coefficient variant Of } \\
\text { (Y) Ycbcr }\end{array}$ & GLCM Homogeneity of I (YIQ) & \\
\hline & $\begin{array}{l}\text { Coefficient variant } \\
\text { Of } \mathrm{L}\left(\mathrm{L}^{*} \mathrm{u}^{*} \mathrm{v}^{*}\right)\end{array}$ & $\begin{array}{l}\square \text { Coefficient variant Of } \\
\mathrm{u}^{*}\left(\mathrm{Lu}^{*} \mathrm{v}^{*}\right) \text {. }\end{array}$ & $\begin{array}{l}\square \text { GLCM Homogeneity of S \& V } \\
\text { (HSV) }\end{array}$ & \\
\hline & $\begin{array}{l}\text { Coefficient variant } \\
\text { Of } u^{*}\left(L^{*} u^{*} v^{*}\right)\end{array}$ & $\begin{array}{l}\text { Coefficient variant Of L } \\
\text { (HSV) }\end{array}$ & GLCM Homogeneity of cr (Ycbcr) & \\
\hline & & & GLCM Homogeneity of L of $\left(\mathrm{Lu}^{*} \mathrm{v}^{*}\right)$ & \\
\hline & & & $\begin{array}{l}\text { Standard deviation of Local range for } \\
\text { L (Lab), }\end{array}$ & \\
\hline & & & GLCM Corrolation of cb of (Ycbcr), & \\
\hline & & & $\begin{array}{l}\text { GLCM Homogeneity of H\& S\&I } \\
\text { (HSI) }\end{array}$ & \\
\hline & & & $\begin{array}{l}\text { GLCM Contrast of } \mathrm{L} \& \mathrm{u}^{*} \text { of } \\
\left(\mathrm{Lu}^{*} \mathrm{v}^{*}\right) \text {. }\end{array}$ & \\
\hline & & & Mean of Local entropy for Y (YIQ) & \\
\hline & & & $\begin{array}{l}\text { Mean of Local entropy for } \mathrm{u}^{*} \\
\left(\mathrm{~L}^{*} \mathrm{u}^{*} \mathrm{v}^{*}\right)\end{array}$ & \\
\hline
\end{tabular}

\section{CONCLUSIONS \& FUTURE WORK}

The proposed non-invasive automated system for PSL and melanoma diagnosis tested upon two different types of superficial skin lesions images. The system was tested on 320 melanocytic skin lesions images, fairly divided within both clinical and dermoscopic images. Computer aided diagnosis systems especially for melanoma begins with image segmentation to extract the infected lesion out of the healthy tissue, this study presented segmentation as first step as well. Then features extraction provides a valuable contribution; it defines the characteristics of the lesion image by illustrating a relevant description for the PSL in a feature space. Features 
categories vary between 91 morphological features based on (geometric, chromatic) and 316 representing texture analyses. Features significance level were evaluated and selected regarding three feature selection methods; f-test, t-test and fisher score ranking. Selected features were fed into three different classifiers; ANN, KNN, SVM. Results obtained was very promising concluded that, this study has the ability to define new and efficient features for melanoma diagnosis. Thus, this study presented an efficient software tool which implements different image processing algorithms developed for the automated analysis of clinical and dermoscopic images.

More specifically, the huge number of experiments performed according to the use of different features categories and different classifiers, to PSL images have been resulted in an integrated architecture for CAD. Experimental results show that integration between the most prominent features selected regarding fisher score ranking from the three efficient features categories; geometric, chromatic and texture features had effectively defined the lesion structure rather than single or dual approaches. Where the combined approach records 96.25 and $97.5 \%$ as the best accuracy resulted upon classifying dermoscopic and clinical images respectively by ANN.

Comparing results achieved through the last multiple model to the recent studies mentioned in the literature review section, this approach considered as promising completed CAD system that add all different features category to cope with the lesion heterogeneous phenomena and diagnose it with accuracy reaches $97.5 \%$.

There are many things that need to be improved upon and worthy of further work. According to segmentation step acquired through that study, tumor edges was smoothed and so important features was rejected, so segmentation process could be improved to have better results. Different feature selection methods could be considered to avoid features redundancy or reputation. Also, the most notable is of course to study the dermoscopic differential structure in a larger scale, which requires a more detailed dermoscopic database.

\section{REFERENCES}

[1] U.S. Emerging Melanoma Therapeutics Market, a090-52, Tech. Rep., 2001.

[2] Ascierto PA, Palmieri G, Celentano E, Parasole R, Caraco C, Daponte A, Chiofalo MG, Melucci MT, Mozzillo N, Satriano RA, Castello G. "Sensitivity and specificity of epiluminescence microscopy: evaluation on a sample of 2731 excised cutaneous pigmented lesions", BJD, pp. 142, 893, 2000.

[3] M. Binder, M. Chwarz, et al., "Epiluminescence microscopy: a useful tool for the diagnosis of pigmented skin lesion for formally trained dermatologists", Archives of Dermatology, vol. 131, pp. 286-291, 1995.

[4] W. Liu, D. Hill, A. Gibbs, M. Tempany, C. Howe, R. Borland, M. Morand, and J. Kelly, "What features do patients notice that help to distinguish between benign pigmented lesions and melanomas? The $\mathrm{ABCD}(\mathrm{E})$ rule versus the seven-point checklist," Melanoma Res., vol. 15, no. 6, pp. 549-554, 2005.

[5] F. Nachbar, W. Stolz, T. Merkle, A. B. Cognetta, T. Vogt, M. Landthaler, P. Bilek, O. Braun-Falco, and G. Plewig, "The ABCD rule of dermatoscopy: High prospective value in the diagnosis of doubtful melanocytic skin lesions," J. Amer. Acad. Dermatol., vol. 30, no. 4, pp. 551-559, Apr. 1994.

[6] Ho Tak Lau, Adel Al-Jumaily. "Automatically Early Detection of Skin Cancer: Study Based on Neural Network Classification|”, International
Conference of SOCPAR, pp. 375-380, 2009.

[7] P. Rubegni, G. Cevenini, M. Burroni "Automated diagnosis of pigmented skin lesions", International Journal of Cancer, Vol. 101, pp. 576-580, 2002.

[8] Iris Cheng, Edwardsville, Illinois, "Skin tumor classification using relative color featuresl". Thesis submitted in partial fulfillment for Master's degree in Electrical Engineering at Southern Illinois University, Edwardsville, Illinois, 2006.

[9] José Fernández Alcón, C`alina Ciuhu, Warner ten Kate, Adrienne Heinrich, Natallia Uzunbajakava, Gertruud Krekels, Denny Siem, and Gerard de Haan, "Automatic Imaging System With Decision Support for Inspection of Pigmented Skin Lesions and Melanoma Diagnosis", IEEE journal of selected topics in signal processing, VOL. 3, pp.14-25, 2009.

[10] Mariam A.Sheha, Mai S.Mabrouk, Amr Sharawy,”Automatic Detection of Melanoma Skin Cancer using Texture Analysis", International Journal of Computer Applications, VOL 42- pp.20, 2012.

[11] Mai S. Mabrouk, Mariam A. Sheha, Amr A. Sharawy "Computer Aided Diagnosis of Melanoma Skin Cancer using Clinical Photographic images", International journal of computer and technology. Vol.10, No.8, May 2013

[12] Mariam A. Sheha, Mai S. Mabrouk, Amr A. Sharawy "Pigmented Skin Lesion Diagnosis Using Geometric and Chromatic Features”, Biomedical Engineering Conference (CIBEC), Cairo International, IEEE, Dec. 2014.

[13] K. Korotkov and R. Garcia, "Computerized analysis of pigmented skin lesions: A review", Artificial Intelligence in Medicine, vol. 56, no. 2, pp. 69-90, 2012.

[14] A.Blum, H.Luedtke, U.Ellwanger, R.Schwabe,* G.Rassner And C.Garbe, "Digital image analysis for diagnosis of cutaneous melanoma. Development of a highly effective computer algorithm based on analysis of 837 melanocytic lesions”, BJD, vol.151, pp.1029-1038, 2004.

[15] Ratner D, Thomas C, Bickers D. "The uses of digital photography in dermatology". Journal of the American Academy of Dermatology, 41(5):749-56, 1999.

[16] www.dermoscopic.blogspot.com/

[17] www.dermoscopyatlas.com

[18] http://www.ee.siue.edu/ sumbaug/Borders\%20Complete/

[19] B. Garcia Zapirain, A. Mendez Zorrilla, I. Ruiz Oleagordia, G. Nunez, A. Abtane,"Skin cancer parameterization algorithm based on epiluminiscence image processing”, IEEE,2009.

[20] www.dermnet.com

[21] Dermnetnz.org

[22] DermQuest.com (www.dermQuest.com)

[23] Dermis.com (dermis.multimedica.de)

[24] dermatlas.med.jhmi.edu

[25] Tim Kam Lee, "Measuring border irregularity and shape of cutaneous melanocytic lesions", thesis submitted in partial fulfillment of the requirements for the degree of doctor of philosophy in the School of Computing Science. Simon Fraser University, January 2001.

[26] Bushra Jalil, Dr Franck Marzani, "Multispectral Image Processing Applied to Dermatology" A Thesis Submitted for the Degree of MSc Erasmus Mundus in Vision and Robotics (VIBOT), 2008.

[27] Tarun Kumar, Karun Verma, "A Theory Based on Conversion of RGB image to Gray Image”, International Journal of Computer Applications (0975 - 8887) Volume 7- No.2, September 2010

[28] G.M. Brake and N. Karssemeijer, 2001. "Segmentation of suspicious densities”, Med. Phys. 28, pp. 258-266.

[29] Otsu N,“A threshold selection method for gray-level histograms, IEEE Trans. Syst., Man, Cyber, vol. SMC-9, pp.62-66, 1979.

[30] Soille, P., "Morphological Image Analysis”, Principles and Applications Springer, pp. 164-165, 1999.

[31] Di Leo G, Fabbrocini G, Paolillo A, et al. "Towards an Automatic Diagnosis System for Skin Lesions: Estimation of Blue-Whitish Veil and Regression Structures", In: Proceedings of the IEEE $6^{\text {th }}$ International Multi-Conference on System, Signals and Devices (SSD '09), Djerba, Tunisia, March 2009. 
[32] Pietro rubegni*, Gabriele cevenini, Marco burroni, Roberto perotti, Giordana dell'eva, Paolo sbano, Clelia miracco, pietro luzi, piero tosi, paolo barbini and lucio andreassi, "Automated diagnosis of pigmented skin lesions”. Publication of the International Union Against Cancer Int. J. Cancer: 101, 576-580 2002 Wiley-Liss, Inc.

[33] Vladimir V, Vassili .S, Alla A, “A Survey on Pixel-Based Skin Color Detection Techniques”, Graphics and Media Laboratory, Moscow state university, Moscow, Russia.

[34] Chun-Liang Chien and Din-Chung tseng, "Color image enhancement with exact HSI color model" . International journal of Innovative computing, Information and control volume 7, number 12 December 2011.

[35] S.K. Tasoulis, C.N. Doukas,I. Maglogiannis, V.P. Plagianakos "Classification of Dermatological Images Using Advanced Clustering Techniques”, 32nd Annual International Conference of the IEEE EMBS Buenos Aires, Argentina, August 31 - September 4, 2010.

[36] R. C. Gonzalez, R. E. Woods, "Digital Image Processing”, 3rd Edition, Addison-Wesley, Reading, MA, USA, 1992.

[37] A. Meyer-Baese, "Pattern Recognition in Medical Imaging", Academic Press, Inc., Orlando, FL, USA, 2003.

[38] Cula O G, Dana K J, Murphy F P, Rao B K. "Skin Texture Modeling”, International Journal of Computer Vision IJVC, 2004.

[39] V. A. Kovalev, F. Kruggel, H.-J. Gertz, D. Y. V. Cramon, "Threedimensional texture analysis of MRI brain datasets", IEEE Transactions on Medical Imaging, 2001.

[40] X. Li, "Texture analysis for optical coherence tomography image", Master's thesis, The University of Arizona, 2001.

[41] Radu Dobrescu, Matei Dobrescu, Stefan Mocanu, Dan Popescu, "Medical images classification for skin cancer diagnosis based on combined texture and fractal analysis", wseas transactions on biology and biomedicine, Issue 3, Volume 7, 2010.

[42] R. M. Haralick, Dinstein, K. Shanmugam, “Textural features for image classification”, IEEE Transactions on Systems, Man, and Cybernetics SMC-3 610-621, 1973.

[43] R. M Haarlick,"Statistical and structural approaches to texture," Proc. IEEE, vol. 67, pp. 786-804, 1979.

[44] J. C. Russ, "The Image Processing Handbook", Raleigh, NC: CRC Press,1995.

[45] Fritz Albregtsen, "Statistical Texture Measures Computed from Gray Level Coocurrence Matrices”, University of Oslo, November 5, 2008.

[46] Gonzalez, R.C., R.E. Woods, S.L. Eddins, "Digital Image Processing Using MATLAB”, New Jersey, Prentice Hall, Chapter 11,2003.

[47] http://www.mathworks.com/help/index.html

[48] Markowski, Carol A; Markowski, Edward. "Conditions for the Effectiveness of a Preliminary Test of Variance", The American
Statistician 44 (4): 22-326,1990.

[49] Sawilowsky, S. "Fermat, Schubert, Einstein, and Behrens-Fisher: The Probable Difference Between Two Means When $\sigma_{1}{ }^{2} \neq \sigma_{2}{ }^{2 n}$, Journal of Modern Applied Statistical Methods, 1(2), 461-472, 2002.

[50] McDonald, J.H., Handbook of Biological Statistics Sparky House Publishing, Baltimore, 2008.

[51] T. Golub, D. Slonim, P. Tamaya, C. Huard, M. Gaasenbeek, J. Mesirov, H. Coller, M. Loh, J. Downing, M. Caligiuri, C. Bloomfield, and E. Lander. "Molecular classification of cancer: Class discovery and class prediction by gene expression monitoring”, Science, 286:531-537, 1999.

[52] Margarita Sordom, "Introduction to Neural Networks in Healthcare", Open Clinical, October 2002.

[53] R.O. Duda, P.E. Hart, and D.G. Stork, "Pattern Classification”, Wiley, 2nd edition, 2001.

[54] A. J. Smola and B. Schoelkopf, "A tutorial on support vector regression”, Neuro COLT2 Technical Report Series NC2-TR-1998-030, ESPRIT working group on Neural and Computational Learning Theory, 1998.

[55] V. Vapnik, "The Nature of Statistical Learning Theory”, Springer, New York, 1995.

[56] B. Scholkopf, S. Kah-Kay, C. J. Burges, F Girosi, P. Niyogi, T. Poggio, and V. Vapnik, "Comparing support vector machines with Gaussian kernels to radial basis function classifiers,” IEEE trans Signal Processing, vol. 45, pp. 2758-2765, 1997.

[57] M. Pontil and A. Verri,"Support vector machines for 3-D object recognition,” IEEE Trans. pattern analysis, Machine Intel, vol. 20, pp. 637-646, 1998.

[58] V. Wan and W.M Campbell, "Support vector machines for speaker verification and identification," in Proc. IEEE Workshop Neural Networks for Signal Processing, Sydney, Australia, pp. 775-784, 2000.

[59] E. Osuna, R. Freund, and F. Girosi, "Training support vector machines: Application to face detection,” in Proc. Computer Vision and Pattern Recognition, Puerto Rico, pp. 130-136, 1997.

[60] B. Schölkopf and A. J. Smola, "Learning with Kernels", MIT Press, 2002.

[61] T. Hastie, R. Tibshirani, J. Friedman, "The Elements of Statistical Learning”, New York, Springer Verlag, 2003.

[62] N. Cristianini and J. Shawe-Taylor, "An Introduction to Support Vector Machines”, Cambridge, U.K, Cambridge Univ. Press, 2000.

[63] R.M. Nishikawa, M.L. Giger, K. Doi, C.J. Vyborny, and R.A. Schmidt, "Computer aided detection of clustered microcalcifications in digital mammograms,”Med. Biol. Eng. Comp. Vol. 33, pp. 174- 178, 1995.

[64] S. Theodoridis and K. Koutroumbas, "Pattern Recognition", Academic Press, San Diego, 1999. 